\title{
7. Learning Analytics an Hochschulen
}

Corinne Büching, Dana-Kristin Mah, Stephan Otto, Prisca Paulicke, Ernst A. Hartman

Digitale Angebote sind inzwischen fester Bestandteil des Bildungssystems. Zahlreiche Hochschulen verwenden Learning-Management-Systems (LMS) zur Unterstützung von Lehre und Studium und bieten immer häufiger OnlineKurse an, z. B. Massive Open Online Courses (MOOC) (Cormier und Siemens 2010). Bei der Nutzung digitaler Lernangebote und Lernumgebungen fallen kontinuierlich Daten an, die sich analysieren lassen, Einblick etwa in das individuelle Lernverhalten geben oder Hinweise darauf, wie die Lehre und das Lernen unterstützt werden könnten, um Lernprozesse zu verbessern. Bislang nutzen Bildungsinstitutionen diese Daten jedoch noch wenig - mittels Learning Analytics könnte sich das ändern. Was aber ist Learning Analytics? Und wie ist es im Kontext Kl einzuordnen?

Learning Analytics verwendet dynamisch generierte Daten von Lernenden, Lehrenden und Lernumgebungen, mit dem Ziel, Lernprozesse und Lernumgebungen zu optimieren (Ifenthaler 2015). Die hierzu verwendeten Daten setzen sich aus leistungs- und personenbezogenen sowie curricularen Variablen - z. B. aktuelle Studienleistungen, Aktivität in universitären Onlinesystemen, soziodemografische Daten zusammen, die u. a. aus dem LMS stammen. Anhand solcher Daten können Lernverhalten analysiert und Lernprofile erzeugt werden. Im Hochschulkontext werden in der Regel Algorithmen verwendet, die aufgrund von Echtzeitdaten berechnen können, wie groß etwa die Wahrscheinlichkeit der erfolgreichen Absolvierung eines Kurses ist, um Risikostudierende zu identifizieren (Arnold 2010). Zudem können die Lernenden automatisch generiertes personalisiertes Feedback zu ihrem Lernprozess sowie individualisierte Empfehlungen zur Unterstützung erhalten (Pistilli und Arnold 2010).

Die individuell angepassten Rückmeldungen, Lernempfehlungen sowie die Vorhersagen zum Lernerfolg (wie die Berechnung von Erfolgswahrscheinlichkeiten durch Learning Analytics ist Gegenstand der Debatte im Kontext von KI, ML, intelligenten Tutorensystemen und adaptivem Lernen (Adams Becker et al. 2017). Adaptives Lernen und Learning Analytics beschrieb bereits 2016 der NMC Horizon Report (Hochschulausgabe), der Technologietrends und Auswirkungen neuer Technologien im Hochschulbereich weltweit erfasst und als Lehr-/Lerntechnologien identifiziert, die innerhalb eines Jahres oder schneller weite Verbreitung finden werden (Johnson et 
al. 2016). Adaptive Lerntechnologien können sich durch große Datenmengen und intelligente Algorithmen immer besser dynamisch und in Echtzeit auf Personen einstellen sowie passende Lernaktivitäten und -inhalte antizipieren (Johnson et al. 2016). Beispielsweise verwendet die Sprachlern-App "Duolingo" virtuelle Chatpartner (Chatbots), die automatisiert und adaptiv auf die Lernfortschritte der Lernenden reagieren können. Auch erste Textbewertungs-Programme für schriftliche Arbeiten, insbesondere in Rahmen von MOOCs, werden entwickelt und erprobt, die auf KIGrundlage den Bewertungsstil der Prüfenden lernen und Texte dementsprechend beurteilen können. Eine vollständige Automatisierung von intelligenten Systemen und Learning Analytics ist jedoch stark umstritten, da bisher menschliche Urteilskraft bei der Leistungsbewertung unabdingbar ist. So zeigt Learning Analytics aggregierte Daten und deren Visualisierung - die Interpretation der Daten sowie die datenevidenten Interventionen sollten anhand lerntheoretischer und pädagogischer Kompetenz durch Lehrende erfolgen.

Aktuell erforschen und nutzen vorrangig englischsprachige Länder wie USA, Australien und das Vereinigte Königreich Learning Analytics. In Deutschland wird es bisher kaum thematisiert und angewendet. Dabei können internationale Studien bereits einen positiven Zusammenhang zwischen dem Einsatz von Learning Analytics und Studienerfolgen aufzeigen (Sclater und Mullan 2017).

\section{Nutzen und Spektrum von Learning Analytics}

Learning Analytics bietet den an Lernprozessen beteiligten Gruppen wie Politik, Institutionen, Instruktionsdesign, Lernende und Lehrende vielfältigen Nutzen (Ifenthaler und Widanapathirana 2014). Beispielweise ermöglicht es auf politischer Ebene institutionsübergreifende Vergleiche und kann als Informationsquelle für Qualitätssicherungsprozesse dienen. Lernende erhalten Einblick in ihre Lerngewohnheiten, können diese reflektieren und optimieren und somit ihre Erfolgschancen erhöhen. Lehrende können ihre Lehrpraktiken analysieren und ihre Lehrqualität verbessern sowie Risikostudierende identifizieren und entsprechend eingreifen (Ifenthaler und Schumacher 2016).

Allgemein geht mit Learning Analytics die Verheißung einher, dass die Analyse der aggregierten Daten und das daraus generierte Feedback an Lehrende wie Lernende die Qualität der Lehr- und Lernprozesse deutlich verbessern kann. Lehrende fragen sich häufig: Welche Wirkung hat meine Lehrveranstaltung erzielt? Konnte ich alle Bedürfnisse der Studierenden einbeziehen? Zumeist wurden und werden diese Fragen durch die Klausurnoten von Studierenden oder durch Lehrevaluationen am Ende des Semesters beantwortet.

Einhergehend mit der technischen Entwicklung hin zu Online- und Blendend Learning-Formaten an den Universitäten sind Studierende hochschulintern mehr und 
mehr online und setzen ihre digitalen Fußabdrücke. Zumeist äußerst sich dies in Form von Online Communities, Foren, Blogs, oder schlicht über eine Lernplattform wie z. B. Moodle der jeweiligen Lehrveranstaltung (Sin und Muthu 2015). Wenn sich Studierende in einem LMS wie Moodle bewegen, eröffnen sich für die Lehrenden neue Möglichkeiten, die digitalen Lerndaten von Studierenden bereits im Lernprozess, also im laufenden Semester, zu verfolgen. Plötzlich sind Daten verfügbar, die den Lehrenden etwa darüber Auskunft geben, wer in welchem Umfang das Lernsetting nutzt, wie etwa Logins, Forenbeiträge. In diesem Zusammenhang spricht man in der anglo-amerikanischen Literatur von dem Wissen über "learning experience in learning environments" (Merceron, Bilkstein und Siemens 2015).

Aus der Sicht eines Lehrenden liegt darin der Vorteil, schon während des Semesters beobachten zu können, welche Lernmedien online besonders gut angenommen werden (z. B. Lehrvideos, Podcasts, Texte), bei welchen Onlinetests die Studierenden vielleicht größere Schwierigkeiten haben oder auch schlicht zu welchen Tages- oder Wochenzeiten sie sich an die Arbeit setzen. Abbildung 7.1 zeigt beispielhaft ein Dashboard, das Lehrenden die Aktivitäten ihrer Studierenden tagtäglich übersichtlich anzeigt und ihnen die Möglichkeit gibt, unmittelbar zu intervenieren, wenn z. B. Studierende unterdurchschnittlich gut mitarbeiten - sei es, dass subjektiv Verständnisschwierigkeiten bestehen, das Lehrmaterial mangelhaft ist oder die Lehrenden etwa den Stoff nicht gut aufbereitet haben.

Anhand des so visualisierten Lernverhaltens der einzelnen Studierenden könnte der Lehrende individuelle Lernwege effektiver erkennen oder unmittelbar Maßnahmen

All Students Activity Report

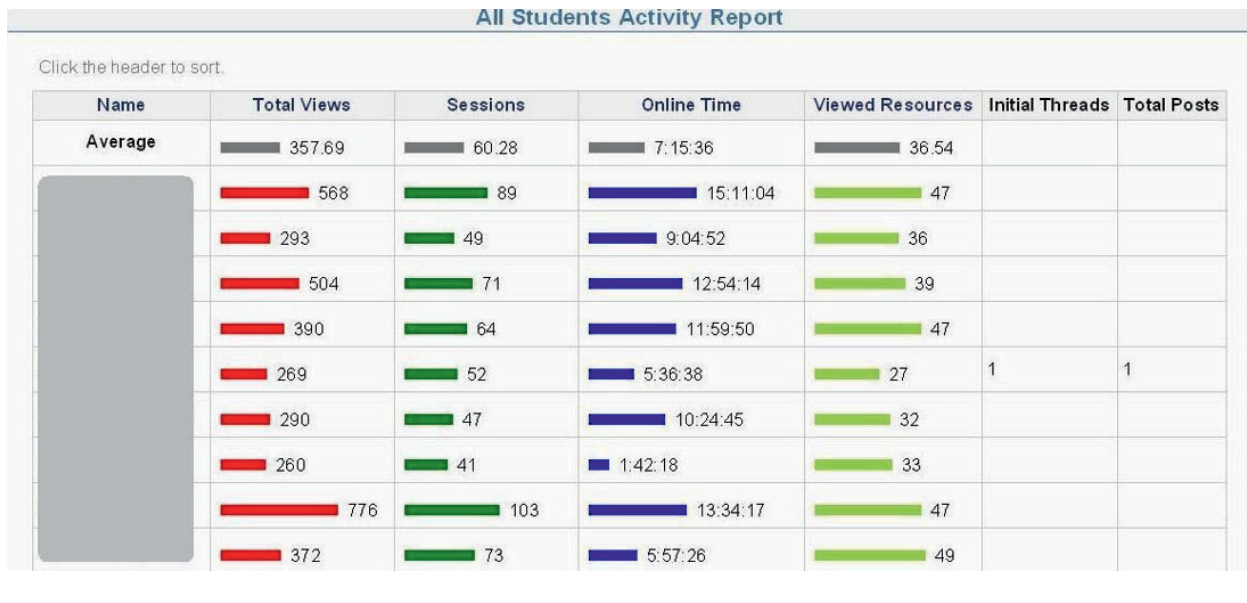

Abbildung 7.1: Ansicht eines Dashboards zu den Aktivitäten von Studierenden (Quelle: Zhang und Almeroth 2010). 
für eine Nachjustierung der Methodik und Didaktik der Lehrveranstaltung treffen. Aus dem skizzierten Bild ergeben sich allerdings Fragen zum Lernen: Welche Erkenntnisse sind durch Learning Analytics im Hinblick auf das Lernverhalten zu erwarten? Ist Learning Analytics die Voraussetzung für adaptives Lernen? Wie können das Lernverhalten oder der individuelle Lernprozess optimiert werden, z. B. durch Verfolgen des Clickstreams bei MOOCs? Liegen Lernschwächen oder besondere Begabungen vor?

Die Daten aus den Lehrveranstaltungen könnten um weitere Studierendendaten ergänzt werden, um sie unter dem Aspekt „Studienerfolg" zu analysieren. Kellen (Kellen et al. 2013) untersuchten die Daten von Studierenden im Zeitverlauf von mehreren Semestern. Sie nutzten Hintergrunddaten (z. B. die Abiturnote) der Studierenden und Daten aus den Lehrveranstaltungen. Sie fügten diese Teilmengen anschließend zu einem Gesamtscore zusammen und konnten erkennen, welche Studierenden positiv auffallen, und sie von denen unterscheiden, die im Mittel hinter ihren Kommilitonen zurückblieben. Zum Einsatz von Learning Analytics als Werkzeug zur Qualitätssicherung und Qualitätsentwicklung ergeben sich folgende Fragen: Kann durch Learning Analytics der Unterricht verbessert werden? Können durch Learning Analytics Risikostudierende identifiziert werden? Ist Learning Analytics also ein geeignetes Instrument zur Vermeidung von Studienabbrüchen? Eignen sich die Daten für Prognosen bzw. kann man aus ihnen künftigen Lernerfolg ableiten?

Auf einer Ebene oberhalb von Lehrveranstaltungen und Studiengängen können Datenanalysen über ganze Bildungsinstitutionen hinweg Aussagen zu deren Effektivität und deren Beitrag zum Studierendenerfolg machen. Allgemein bekannt sind hier die PISA-Studien (Programme for International Student Assessment).

Zusammenfassend kann festgehalten werden, dass unterschiedliche Gruppen von Beteiligten (z. B. Studierende, Dozierende, Hochschulleitungen oder auch Regierungsinstitutionen) unterschiedliche Interesse an Daten haben können, die etwas über den Studierendenerfolg auf Mikroebene einer Lehrveranstaltung, auf Mesoebene der Studiengänge oder Makroebene der Hochschulen aussagen (siehe Abbildung 7.2).

Mögliche Ziele von Learning Analytics sind nachfolgend in Anlehnung an Leitner und Ebner (2017) aufgelistet:

- Identifizierung und Unterstützung von Risikostudierenden

- Verbesserung von Retention und Leistung

- Visualisierung der Lernleistung anhand einer Vergleichsgruppe

- (Echtzeit-)Feedback zu Lernperformance und -aktivität 
- Verbesserung der Gruppenarbeit durch Aufzeigen der Mitwirkung der einzelnen Gruppenmitglieder

- Visualisierung zum Engagement und Niveau in Diskussionsforen

- Förderung der Reflexion und Selbsteinschätzung im Lernprozess

- Aussagen zur Interaktion in einem E-Learning-System

- Evaluation von Lehr- und Lernprozessen

Ziele von Learning Analytics, Qualitäts- und Evaluationskriterien sowie Chancen und Herausforderungen sind in einer Vielzahl von Frameworks formuliert, die als Richtlinien für die erfolgreiche Entwicklung, Implementierung und Anwendung von Learning Analytics fungieren sollen (Greller und Drachsler 2012; Scheffel et al. 2015). So ist die Erhöhung des Studienerfolgs ein zentrales Ziel von Learning Analytics und im Erfolgsfall auch deshalb interessant, weil so im Umkehrschluss hohe Studienabbruch-

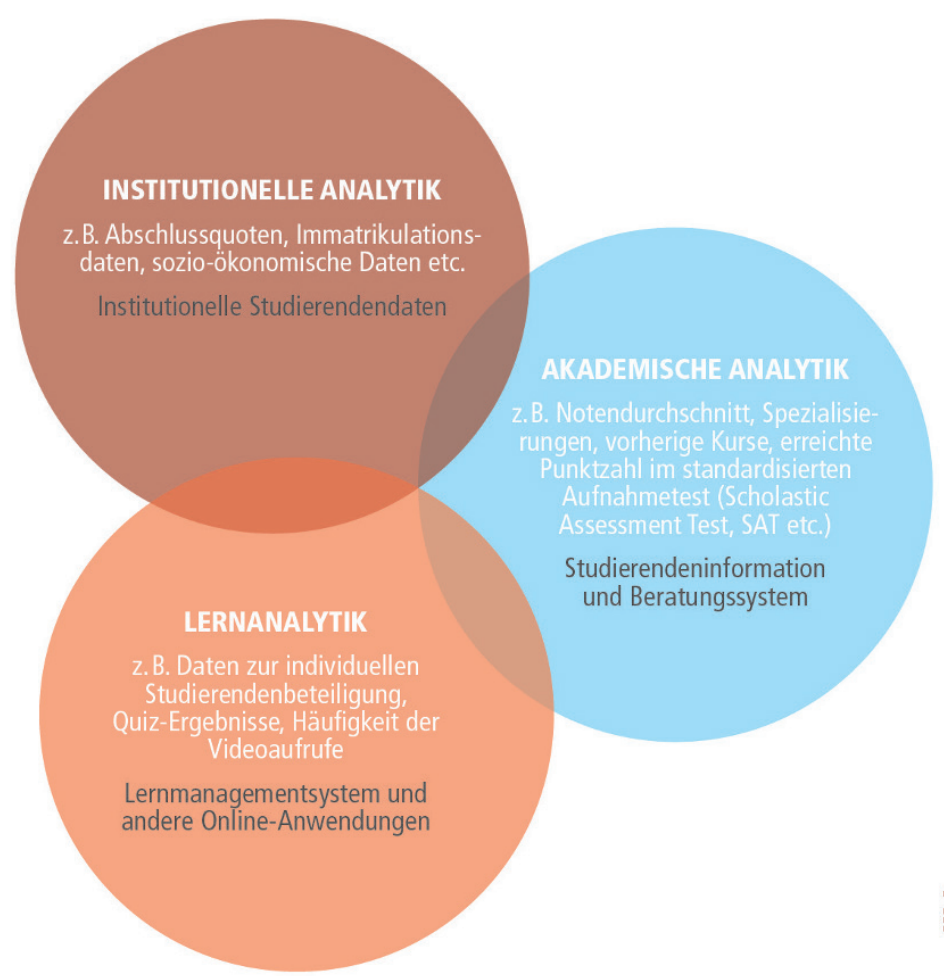

ำ:"

Abbildung 7.2: Dimensionen der Analyse von studentischem Erfolg (Quelle: Elias 2011, eigene Darstellung und Übersetzung). 
quoten vermeidbar werden (Mah et al. 2016). Deutschland strebt die Erhöhung von Studienerfolg an, um beispielsweise dem Fachkräftemangel zu begegnen (BMBF 2012). Den Studienerfolg vorherzusagen und Risikostudierende zu identifizieren ist auf Basis von leistungs-, personenbezogenen und curricularen Variablen möglich (Arnold und Pistilli 2012). Studierende erhalten personalisiertes Feedback zu ihrem Lernprozess auf Kursebene sowie Empfehlungen für Unterstützungsangebote (Pistilli und Arnold 2010). Diese Informationen werden den Nutzern nahezu in Echtzeit im Dashboard zur Verfügung gestellt, beispielsweise visualisiert als Ampel oder Kompass (Verbert, Duval, Klerkx, Govaerts und Santos 2013).

\section{Tools und Techniken}

Immer dann, wenn Studierendendaten einem kontinuierlichen Tracking bzw. einem systematischen Assessment unterzogen werden sollen, kommen zumeist Contentoder Learning-Management-Systeme (CMS/LMS) zum Einsatz. Diese sind nach Hijon und Carlos (2016) von einer umfassenden und zufriedenstellenden Funktionalität

\section{Overall Summary of User Activity}

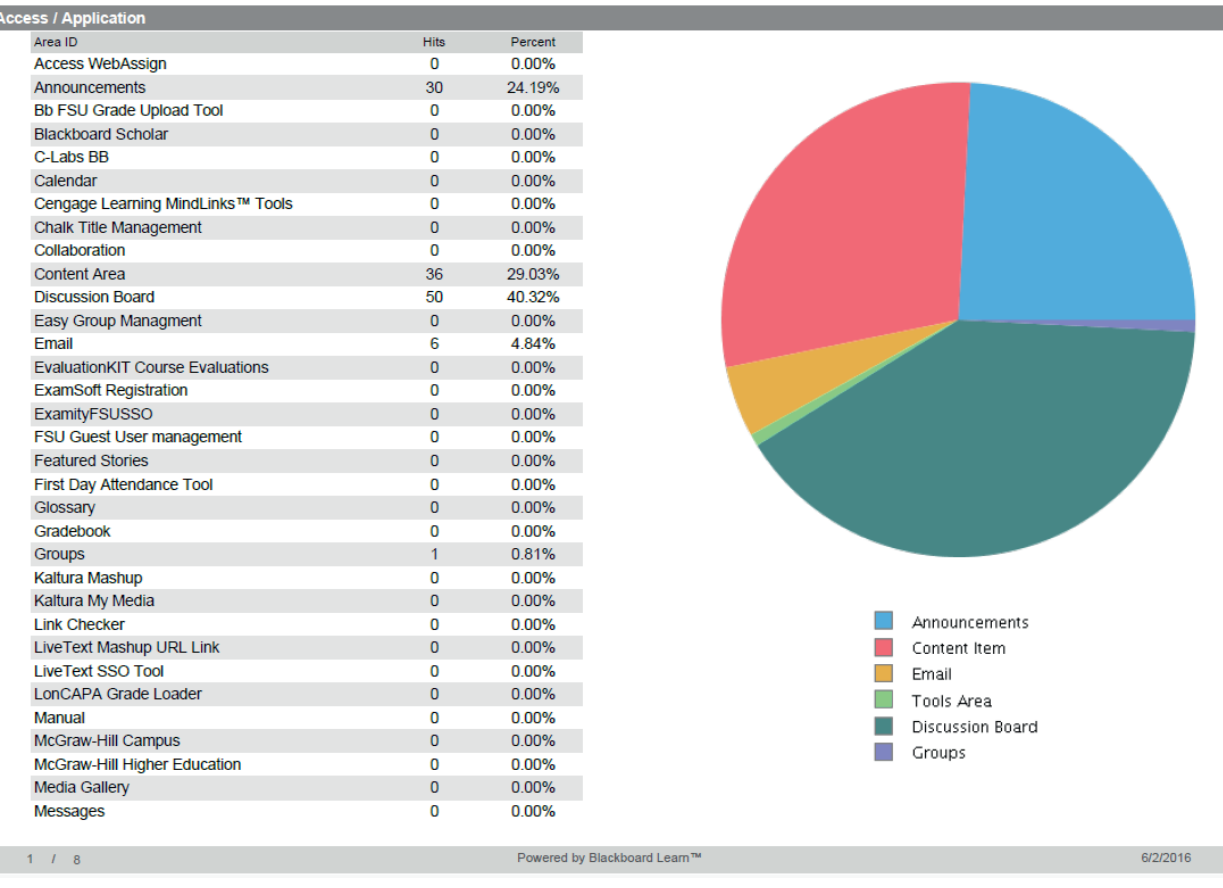

Abbildung 7.3: Ansicht eines Kursberichts über die Aktivitäten der Studierenden (Quelle:

Florida State University 2018). 
noch weit entfernt. Auf der Ebene einer Lehrveranstaltung werden unter der Überschrift Learning Analytics vor allem Häufigkeiten der Aktivitäten von Studierenden erhoben, z. B. Interaktion zwischen innen und angebotenem Lernmaterial oder unter Kommilitonen. Ein mögliches Interface könnte in Anlehnung an Abbildung 7.3 gestaltet sein.

Je nach Fragestellung können jedoch auch Netzwerkanalysen, die den Prozess der Interaktion von Studierenden aufzeigen, in einem Netzdiagramm visualisiert werden (siehe Abbildung 7.4).

Wenn Einflüsse zwischen Noten, Modulen und Studiengängen untersucht werden sollen, spielen neben den genutzten LMS (z. B. Moodle, ILIAS) auch die CMS der Hochschulen, insbesondere diejenigen, die das Prüfungsamt nutzt, eine zentrale Rolle. Wenn bedeutende Variablen von Studierendengruppen (z. B. Geschlecht, Abschluss, Migrationsgeschichte) sowie einzelner Studierender zusammengeführt und analysiert werden könnten, ließen sich einige interessante Fragen beantworten. Hochschulen könnten auf diese Weise $u$. a. ihre Zulassungsverfahren überprüfen,

\section{Social Networks Adapting Pedagogical Practice (SNAPP)}

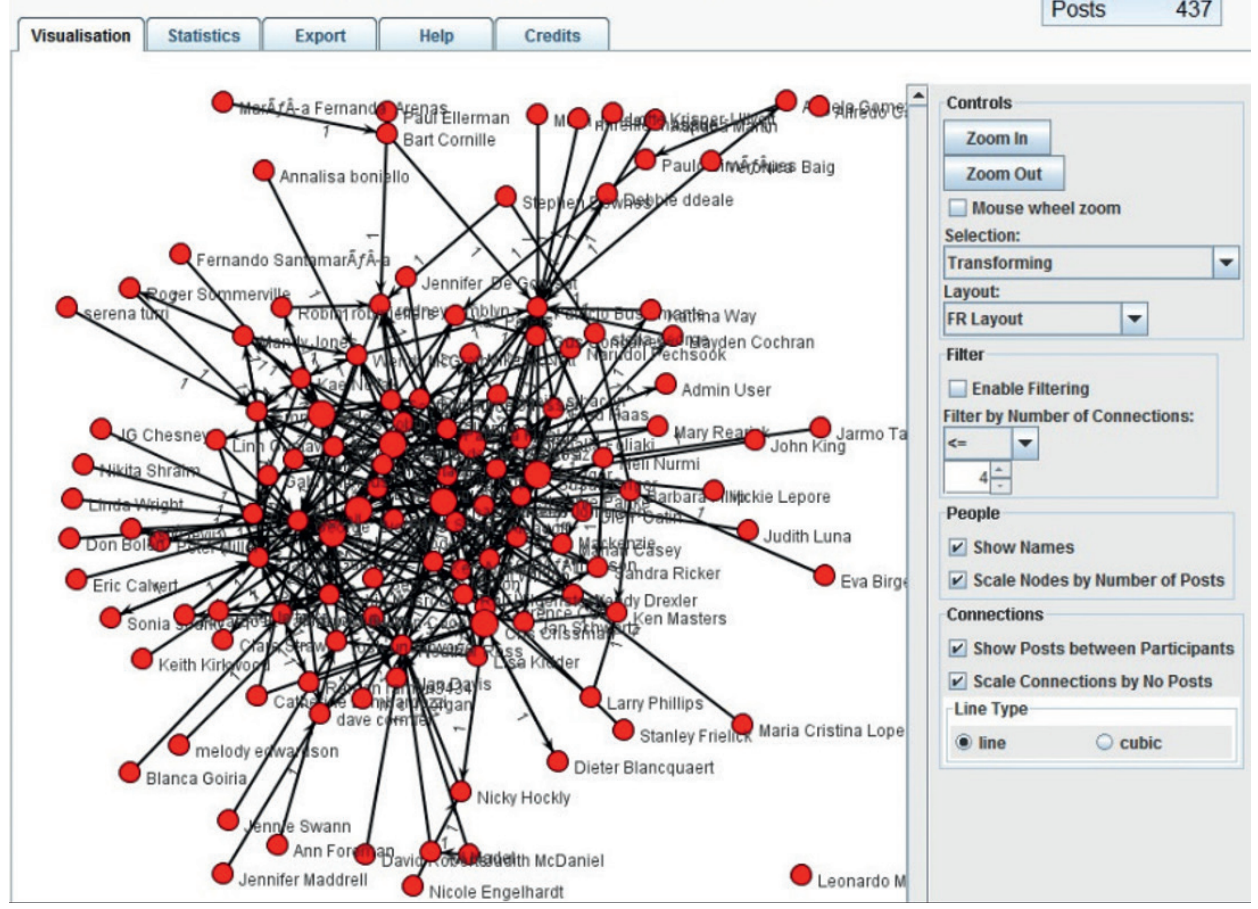

Abbildung 7.4: Visualisierung einer Analyse sozialer Netzwerke (Quelle: Panke 2010). 
etwa: Lassen wir die richtigen Studierenden in einem bestimmten Studiengang zu? Was braucht ein Studieninteressierter, um das Studium der Wirtschaftswissenschaft in unserer Institution erfolgreich zu absolvieren? Welche Teilgruppen profitieren besonders von welcher Art von Unterstützung?

Das Zusammenführen einzelner Variablen kann für die Beantwortung solcher Fragen neue Möglichkeiten bieten. Kritiker geben vor dem Hintergrund aktueller Datenschutzdebatten zu bedenken, dass die Freigabe von persönlichen Daten für den eigenen Lernprozess (z. B. Feedback vom Dozierenden) oder für einen höheren Zweck (z. B. um nachfolgenden Studierenden effektive Lernsettings zu bieten) kontrovers diskutiert wird.

\section{Praxisbeispiele im Hochschulkontext}

Während im angloamerikanischen Raum bereits eine Vielzahl von entsprechenden Programmen an den Universitäten implementiert ist - und deren Wirksamkeit erforscht wurde - steckt die Anwendung von Learning Analytics im deutschsprachigen Hochschulbereich noch in den Kinderschuhen (Sclater, Peasgood, Mullan 2016). Allerdings gibt es hierzulande zunehmend Bestrebungen, innovative Szenarien auf Grundlage von Learning Analytics zu identifizieren und deren Potenziale für die deutsche Hochschullandschaft nutzbar zu machen (Ifenthaler, Mah und Yau 2017). Der konkrete Einsatz von Learning Analytics für spezifische Zielsetzungen lässt sich exemplarisch an drei Projekten aufzeigen:

\section{Early Alert an der University of New England, Australien}

Die University of New England, New South Wales in Australien, hat mehr als 18.000 Studierende, von denen viele das Studium in Teilzeit absolvieren und als Nicht-traditionelle Studierende gelten. Vor dem Einsatz von Early Alert lag die Studienabbruchsquote bei etwa 18 Prozent, was die Hochschule nicht länger hinnehmen wollte (Sclater, Peasgood, Mullan 2016, S. 33). Aus diesem Grund richtete sie Early Alert als mehrschichtiges Learning-Analytics-System mit dem Ziel ein, das Befinden der Studierenden und deren individuelle Lehr-Lern-Bedürfnisse zu ermitteln. Neben einem Abfrageportal (e-Motion), in dem Studierende online ihre aktuelle Befindlichkeit über Emoticons melden können, haben sie im Early-Alert-System zusätzlich die Möglichkeit, textbasierte Rückmeldungen über The Vibe zu geben und zugleich auch die ihrer Kommilitonen einzusehen. Alle Studierendendaten werden in der Automated Wellness Engine (AWE) zusammengeführt, in der diese zusätzlich um Daten zu "class attendance, previous study history, prior results, assignment submissions, and access patterns for the student online portal and other university websites" (Sclater, Peasgoog, Mullan 2016, S. 33) ergänzt werden. Klassifiziert nun das System die Entwicklung eines Studierenden als problematisch, wird dieser zunächst per E-Mail angesprochen und angefragt, ob 
Unterstützung benötigt wird. Hilfestellungen werden dann - je nach Notwendigkeit - um Telefon- oder persönliche Beratung ergänzt. Das Early-Alert-System konnte dazu beitragen, dass nur noch 12 Prozent ihr Studium abbrachen.

\section{MentOR an der Universität Duisburg-Essen}

Das Programm MentOR (Mentoring im Orientierungspraktikum mit online-gestützter Rückmeldung) ist ein Projekt an der Fakultät für Bildungswissenschaften, das sich explizit an Lehramtsstudierende in der Studieneingangsphase richtet und ihnen auf der Basis unterschiedlicher Fremd- und Selbsteinschätzungen eine Rückmeldung zur Kompetenzentwicklung und zur Eignung für den Lehrerberuf bietet (Niemann et al. 2014). Rund um das erste Schulpraktikum im Bachelor-Lehramtsstudium geben die Studierenden eine Selbsteinschätzung zu ihren Kompetenzen vor und nach dem Praktikum ab, die um die Fremdeinschätzungen der Lehrkräfte im Praktikum und der Dozierenden des universitären Begleitseminars auf einer Online-Plattform ergänzt werden.

Die so generierten individuellen Daten von den Studierenden werden diesen dann in individuellen Kompetenzprofilen zur Verfügung gestellt. Im Rahmen von Rückmeldegesprächen mit dem Dozierenden des Begleitseminars ermittelt dieser gegebenenfalls daraus abgeleitete Optionen für eine Beratung (Niemann et al. 2014).

\section{Die Learning Analytics-Strategie an der Open University im Vereinigten Königreich}

Die Open University ist die größte staatliche Universität in Großbritannien und Europa und basiert als Fernuniversität auf dem Supported Distant Learning System, welches einen ortsunabhängigen Zugriff auf sämtliche Studieninhalte ermöglichen soll.

Schon diese Struktur der Universität lässt auf einen möglichen Bedarf an Feedbackformaten auf Basis von Learning Analytics schließen, um Lehr- und Lernsettings effektiv zu gestalten. Anders als viele Hochschulen, die Learning Analytics im Rahmen eines Projekts mit einem spezifischen Fokus (Zielgruppe, Fakultät) nutzen, ist Learning Analytics an der Open University in eine umfassende Gesamtstrategie eingebunden, die eine Vielzahl von Projekten mit fachspezifischem und fachübergreifendem Fokus beinhaltet. So wird u. a. das wöchentliche Arbeitspensum der Studierenden an der Open University erfasst. Weiterhin erhebt eine Längsschnittstudie online an drei Universitäten die Kompetenzentwicklung der Studierenden, um hieraus Bedingungen für das Gelingen von Lehre und Lernen abzuleiten. Wie viel Peer-Unterstützung Studierende brauchen, wird mit qualitativen Verfahren (social network analysis, Lerntagebücher, Fokusgruppeninterviews) ermittelt. Die Forschungsergebnisse auf Grundlage der über Learning Analytics erhobenen Daten verweisen darauf, welches Feedback Studierende bevorzugen (Nguyen et al. 2016), oder auf die möglichst lerneffektive Bereitstellung von Selbstlernmaterial (Toetenel und Rienties 2016). 


\section{Herausforderungen und Risiken}

Den vielfältigen Chancen von Learning Analytics stehen Herausforderungen und Risiken gegenüber wie Datenqualität, Datenschutz und Privatsphäre sowie Akzeptanz der Nutzergruppen (Pardo et al. 2014; Slade und Prinsloo 2013). Zunächst gibt es grundsätzlich die Schwierigkeit, die anfallende Menge an unstrukturierten Daten und Metadaten zu organisieren. Educational Data Mining ist eine Möglichkeit, die Daten für weitere Analysen zugänglich zu machen. Learning Analytics hat wiederum die Aufgabe, die Daten zu analysieren und zu visualisieren. Die Datenqualität ist aber unterschiedlich und die Warnung vor wissenschaftlich nicht begründeten Schlussfolgerungen über Lehr- und Lernprozesse wird laut. Zudem besteht die Gefahr, dass Wissen über Lehr- und Lernprozesse aufgrund von statistischen Wahrscheinlichkeiten gewonnen wird, Learning Analytics die Daten jedoch nicht interpretiert und somit wissenschaftlich unvollständig begründete Zusammenhänge hergestellt werden (Loser 2016). Learning Analytics fragt beispielsweise nicht nach den Gründen für schlechte Antworten, sondern zeigt statistisch auf, dass eine Lernschwäche bei bestimmten Antwortmustern vorhanden ist. Das sollte der Wissenschaft nicht genügen, so Loser (2016). Die Interpretation der Daten erfolgt durch menschliche Beurteilung. Diese kann sich mittels Learning Analytics auf eine Vielzahl von Daten stützen und daraus datenevidente Interventionen ableiten. Es handelt sich um eine sinnvolle Zusammenarbeit von Mensch und Maschine, um Lehr-/Lernprozesse und Lernumgebungen zu optimieren.

Aus der Perspektive der Studierenden wird häufig in populärwissenschaftlichen Debatten vor dem "gläsernen Studierenden" gewarnt. Tatsächlich konnte man noch nicht abschließend und zufriedenstellend klären, wie die Studierenden-Daten anonym bleiben können. Zudem ist fraglich, ob Studierende einen derart umfassenden Überblick über ihre Daten haben, um eine bewusste Entscheidung über Teilnahme und Datenfreigabe für die Analyse treffen zu können. Damit einhergehend ist Freiwilligkeit ein Thema, da für manche Angebote ein Zwang zur Nutzung (z. B. von E-Learning-Plattformen) besteht. Eine weitere Gefahr für Lernende besteht in einer möglicherweise entstehenden „Filter-Blase": Die Prognose von Verhaltenswahrscheinlichkeiten und personalisierte Empfehlungen begrenzen die Entwicklungsmöglichkeiten mit den Konsequenzen, dass die Kreativität unterdrückt werde sowie falsche Pfade und Scheitern, die auch Quelle des Lernens darstellen können, unwahrscheinlicher werden (Loser 2016). Denkbar sei in diesem Zusammenhang, dass wirtschaftliche und politische Interessen die Vorschläge beeinflussen, die das jeweilige System macht.

Die Gefahren für die Lehrenden an Hochschulen können aus zwei Perspektiven betrachtet werden. Erstens aus der Sicht auf die Kompetenz der Lehrenden selbst und zweitens aus der Perspektive der Lehrenden auf die Studierenden und deren 
Bewertung. Betrachtet man die Kompetenz der Lehrenden, lässt sich derzeit festhalten, dass diese hinsichtlich der Möglichkeiten und Tools, die Learning Analytics bietet, besser qualifiziert sein sollten. Anders formuliert: Um Learning Analytics und KI zielführend und gewinnbringend für Hochschullehrende einzusetzen, müssen Qualifikationsformate erarbeitet und angeboten werden. Was die Beziehungen von Lehrenden zu Studierenden betrifft, bestehen die Gefahren der Kontrolle, der Stereotypisierung sowie der Leistungsbewertung durch nicht-menschliche Akteure.

Für die deutschen Hochschulen lässt sich bei einer Etablierung von Learning Analytics eine Herausforderung formulieren: Es handelt sich um die „adäquate Interpretation der Daten, da Learning Analytics zwar mit dem Engagement und der Beteiligung von Studierenden korreliert, aber noch keine Aussage darüber trifft, was Lehrende verändern sollten" (Ionica 2016). Damit wird angesprochen, dass sehr viele Daten angehäuft, weniger analysiert und noch weniger in die Praxis als konkrete Umsetzung rückgekoppelt werden. Was passiert mit den Daten, zu welchem Zweck werden sie gesammelt und unter welchen Gesichtspunkten werden sie analysiert? Das sind einige der Fragen, denen sich die Hochschulen stellen müssen. Vor dem Hintergrund, dass der Markt von Learning-Analytics-Tools stark fragmentiert ist und derzeit dringend Normen notwendig sind (Leitner et al. 2017, 380ff.), werden Standards und Datenaustauschformate immer wichtiger, die einen sicheren Transfer sensibler Daten gewährleisten. Konkret: Die Hochschulen müssen das Datenmanagement klären und einer Überwachungskultur entgegentreten.

Der Umgang mit Bildungsdaten ist bislang weitestgehend nicht reguliert, IT-Sicherheitsstandards fehlen. Datenschutzbeauftragte warnen vor den Gefahren von Big Data. Wirtschaftliche, pädagogische und ethische Bedenken über Konsequenzen werden laut, und es wird formuliert, dass gegenwärtig kaum abzuschätzen sei, welche Risiken mit Learning Analytics und KI im Hochschulbereich einhergehen (Jülicher 2015, S. 3).

Es bleibt festzuhalten, dass die Interpretation von Daten mit Learning Analytics im Hochschulbereich vor Herausforderungen steht, "die noch viel Forschung, Beratung und eine Qualifizierung für Studierende und Hochschullehrende voraussetzt. Vor allem muss das Vertrauen aller Beteiligten in die Transparenz und Sicherheit der Daten und der Prozesse hergestellt werden, auch wenn die dafür notwendigen Technologien insbesondere für die Datensicherheit längst vorhanden sind" (Ionica 2016).

\section{Zukunftsperspektiven zum Einsatz von Learning Analytics mit KI-Bezug}

Nach der Darstellung von Learning Analytics im Hochschulkontext hinsichtlich Nutzen, Tools und Techniken, Praxisbeispielen sowie Herausforderungen, sollen anknüpfend an aktuelle Projekte und Entwicklungen, nachfolgend drei Zukunftsperspekti- 
ven zum Einsatz von Learning Analytics mit KI-Bezug skizziert werden: (1) Personalisiertes Lernen, (2) automatisiertes Feedback und Beratung sowie (3) humanoide Roboter als Assistenten in der Hochschullehre.

1. Personalisiertes Lernen. Schon jetzt liegen zahlreiche elektronische Lernendendaten vor, die durch das verstärkte online Lernen und online Lehren sowie durch die Digitalisierung von Lehr- und Lernumgebungen (z. B. MOOCs, LMS) noch zunehmen werden. Mit Learning Analytics werden diese Daten verarbeitet, analysiert und visualisiert, um Lehrende eine datengestützte Grundlage zur Einschätzung von Lernenden und Ableitung von Unterstützungsmaßnahmen zu bieten. Zudem ermöglicht Learning Analytics Einblicke in Lernverhalten und Lernfortschritte, wodurch Lernund Lehrumgebungen optimiert und Lernen personalisiert werden könnte.

Die Personalisierung von Lerninhalten ist Ziel des Projekts "HyperMind - Das antizipierende Physikschulbuch"48 der Technischen Universität Kaiserslautern in Zusammenarbeit mit dem Deutschen Forschungszentrum für Künstliche Intelligenz (DFKI), gefördert vom Bundesministerium für Bildung und Forschung. Entwickelt werden soll ein intelligentes Physikschulbuch, das adaptiv-dynamisch Inhalte und Aktivitäten entsprechend der individuellen Kompetenzen und Bedürfnisse der Lernenden zur Verfügung stellt. Grundlage für die Aktivitätserkennung ist ein Eye-Tracker, der unter dem Display des Schulbuchs (Tablet oder Computerbildschirm) angebracht ist und die Blickposition der Lernenden erfasst. Somit wird die Verweildauer des Blicks auf den unterschiedlichen Abschnitten - z. B. Einleitung, Definitionen, Anwendungsbeispiele - während der verschiedenen Testphasen - z. B. Textlesen, Aufgabenlösen - gemessen. Diese Daten werden mit KI-Algorithmen analysiert, um Unter- und Überforderung, Lernverhalten und -fortschritte sowie Präferenzen und Lernniveaus vom Anfänger über Fortgeschrittene bis zu Expertinnen und Experten zu untersuchen.

Zukünftig vorstellbar wäre eine verstärkte Verknüpfung von Learning Analytics mit diversen Sensordaten wie Eye-Tracking für Blickposition oder Smartwatches für Pulsmessung, um vertiefte Einblicke in Lernendenverhalten zu erhalten, automatisiert adaptiv-dynamische Lerninhalte zu generieren und somit personalisiertes Lernen zu ermöglichen. Perspektivisch wäre dieses Szenario zur automatisierten Unterstützung personalisierten Lernens in verschiedenen Bildungskontexten - z. B. Schule, Hochschule und Weiterbildung - denkbar.

2. Automatisiertes Feedback und Beratung. Persönliches Feedback zum Lernprozess ist für Lernende sehr wichtig - im Hochschulkontext insbesondere im ersten Studienjahr, der sogenannten Studieneingangsphase, in dem die meisten Studienabbrüche

48 https://www.physik.uni-kl.de/en/kuhn/forschungsprojekte/aktuelle-projekte/uedul hypermind/, zuletzt geprüft am 22.06.2018 
stattfinden (Heublein et al., 2017). Bei der großen Anzahl Studierender ist ein individuelles Feedback durch einen Lehrenden aus Kapazitätsgründen schwer realisierbar - genau hier könnte eine KI eingesetzt werden. Wie einleitend beschrieben, ist ein Ziel von Learning Analytics das Feedback zu Lernperformance und -aktivität in Echtzeit. Das Course-Signals-System der Purdue University (USA) zeigt Studierenden und Lernenden Erfolgswahrscheinlichkeiten auf Kursebene anhand eines Ampelsystems an - grün: hohe Erfolgswahrscheinlichkeit, gelb: mittlere Erfolgs-/Misserfolgswahrscheinlichkeit, rot: hohe Misserfolgswahrscheinlichkeit (Pistilli und Arnold 2010). Feedback in Echtzeit mittels Learning Analytics gewinnt an Bedeutung, da das Angebot an Online-Kursen zunimmt, wenngleich Abbruchraten bei Online-Kursen besonders hoch sind. Auch in Online-Kursen sollten Lernende möglichst frühzeitig und regelmäßig Feedback zu ihren Lernfortschritten erhalten. In diesem Rahmen sind Beratungsangebote, wie beispielsweise allgemeine Studienberatung, Studienorientierung und Mentoring wichtige Aspekte eines Studiums.

Die Kiron Open Higher Education gmbH ${ }^{49}$ bietet seit 2015 ein Online-Studienprogramm für Geflüchtete an, um gleitende Zugänge zur Hochschulbildung zu ermöglichen. In Kooperation mit derzeit 56 Partnerhochschulen bietet Kiron ein „Blended Learning 2.0"-Bildungsmodell an. Bis zu zwei Jahre kann ein Onlinestudium von den geflüchteten Personen absolviert werden, daran anschließend erfolgt an einer Partnerhochschule zwei Jahre lang ein Präsenzstudium. Derzeit sind mehr als 3.300 Studierende auf der Kiron-Plattform registriert. Neben den fünf Fachbereichen (Business and Economics, Mechanical Engineering, Computer Science, Political Science und Social Work) werden Beratungs- und Unterstützungsangebote im Rahmen eines Beratungsnetzwerks offeriert. Um die Maßnahmen in der Orientierungsphase möglichst effizient zu gestalten, werden digitale Beratungsangebote eingesetzt, wie ein Self-Assessement-Tool zur Entscheidungsfindung, ein Onboarding-MOOC für zugelassene Studierende zur Information über erste Schritte auf der Kiron-Lernplattform und Live-Online-Sessions. Perspektivisch sollen durch vermehrte Automatisierung noch mehr Menschen mit digitalen Angeboten erreicht werden.

Zukünftig denkbar wären hier automatisierte personalisierte Beratungsangebote auf Basis von aufbereiteten Lernendendaten mittels Learning Analytics. Hierbei wäre es vorstellbar, dass KI-Algorithmen aus den existierenden Daten lernen, Muster zu erkennen und Beratungsbedarfe klassifizieren. Eine Automatisierung von Betreuungs- und Beratungsprozessen mittels KI-Einsatz, beispielsweise bei Standardfragen, würde mehr Freiraum für individuelle Gespräche und Beratung durch Dozierende und Beratungspersonal ermöglichen.

49 https://kiron.ngo/wp-content/uploads/2017/02/2017-09-25_INTEGRAL\%C2\%B2_ Broschu\%CC\%88re_Abschlussveranstaltung.pdf, zuletzt geprüft am 13.07.2018 
3. Humanoide Roboter als Assistenten in der Hochschullehre. Die Digitalisierung der Hochschulbildung erfordert neue digitale Lehr- und Lernszenarien. Die Methode "Inverted Classroom" (auch "Flipped classroom" genannt) bezeichnet die Methode des umgedrehten Unterrichts. Lehrinhalte werden digital in Form von MOOCs, Lernvideos, Lernmaterialien und Tests auf Onlineplattformen zur Verfügung gestellt und ortsunabhängig eigenständig zur Vorbereitung auf die Präsenzveranstaltung erarbeitet. Die Präsenzveranstaltung wird für Vertiefungen, gemeinsames üben, diskutieren und kollaboratives Arbeiten genutzt. Kollabroatives Arbeiten ist jedoch betreuungsintensiv - und mit wenig Lehrenden und vielen Studierenden eine Herausforderung. Wie könnten hier humanoide Roboter (charakterisiert durch menschliche Form und menschliches Verhalten) als Assistenten die Lehrenden unterstützen? Genau diese Frage wird in dem vom Bundesministerium für Bildung und Forschung geförderten Projekt "H.E.A.R.T. (Humanoid Emotional Assistant Robots in Teaching)" 50 an der Philipps-Universität Marburg erforscht. Der humanoide Roboter "Pepper" ${ }^{41}$ wird als Assistent in Lehrveranstaltungen an der Philipps-Universität Marburg eingesetzt, z. B. um Aufgaben zu stellen, Quizze durchzuführen und auf Fragen der Studierenden zu antworten. Pepper ist auf menschliche Aktionen und Reaktionen programmiert und somit prädestiniert für Mensch-Maschine-Kommunikation und Mensch-Maschine-Beziehung. Technisch möglich ist dies durch maschinelle Spracherkennung (mehrere Sprachen), Sprachsynthese, natürlich-sprachlicher Dialogfähigkeit, verbale Kommunikation über Gestik, Blickkontakt und Körperhaltung sowie Gesichtserkennung. Das eingebaute Tablet ermöglicht zudem den Einsatz für Lehr- und Präsentationsaufgaben.

Perspektivische Anknüpfungspunkte zu Learning Analytics könnten der Einsatz humanoider Roboter als individuelle Lern- und Beratungsassistenten auf Basis der analysierten Lernendendaten sein (z. B. aus den Online-Tests zur Vorbereitung auf die Präsenzveranstaltung) oder die persönliche Unterstützung von automatisch identifizierten Studierendengruppen (z. B. hinsichtlich Interesse und Lernniveau) auf elektronisch verfügbarer Datengrundlage.

\section{Fazit und Ausblick}

Learning Analytics birgt ein großes Potenzial zur Optimierung von Lehr- und Lernprozessen, denn datenbasierte Erkenntnisse bieten vielfältige Vorteile für alle Beteilig-

50 https://www.project-heart.de/, zuletzt geprüft am 13.07.2018

51 https://cdn.website-start.de/proxy/apps/a1 tvb2/uploads/gleichzweilinstances/3A26FA88E7D9-41CA-BB2F-DBE354A2A784/wcinstances/epaper/a735bb42-2925-4a06-be471a2f50374bee/pdf/heart_peppers_f\%C3\%A4higkeiten_broschuere_gro\%C3\%9F.pdf, zuletzt geprüft am 13.07.2018 
ten. Für Hochschulen ist Learning Analytics interessant und vielversprechend, um Risikostudierende frühzeitig zu identifizieren und Studienerfolg zu fördern. Wie deutsche Hochschulen von internationalen Erfahrungen mit Learning Analytics profitieren können, wird derzeit erforscht (Ifenthaler et al. 2017). Für die Bewertung der Ergebnisse eignen sich die bereits existierenden Projekte wie MentOR an der Universität Duisburg-Essen. Dennoch bleibt festzuhalten: In Deutschland steht der Einsatz von Learning Analytics noch am Anfang. Das wiederum bietet "die Chance - frühzeitig und im Dialog mit allen Beteiligten - tragfähige Strategien im Umgang mit der Verknüpfung, Auswertung und Analyse von Bildungsdaten zu entwickeln" (Jülicher 2015, S. 3).

Abschließend ist zu betonen, dass die Zukunft von Learning Analytics in einer fundierten, ganzheitlichen Betrachtungsweise durch interdisziplinäre Ansätze und Forschungsdisziplinen liegt, die nicht nur Häufigkeiten (z. B. Logins im LMS, Anzahl von Foreneinträgen und Gruppendiskussionen, fristgerechte Bearbeitung von Assessments) zählen, sondern diese mit Inhalten verknüpfen. Die Verbindung von Lehr- und Lernforschung, Informatik und Statistik sowie die Einbindung einer ethischen Perspektive sind folglich essentiell (Ifenthaler und Schuhmacher 2016). Wichtige Fragen beziehen sich vor allem auf den Datenschutz und die Privatsphäre, den transparenten Umgang mit Learning Analytics und Informationen für alle beteiligten Nutzergruppen. Hierbei sollte verstärkt die Interpretation der Daten im Fokus stehen, die von den menschlichen Nutzern zu leisten ist. Die Relevanz menschlicher Präsenz und Urteilskraft ist somit elementar im Bildungskontext, wenngleich die Datenaufbereitung mittels Learning Analytics sowie zukünftige Entwicklungen mit KI-Bezug Lehren und Lernen unterstützen können. Perspektivisch denkbar ist die Verarbeitung von Lernendendaten auf Basis von deep learning (siehe Einleitung zu Kapitel Technologie „Entwicklungswege zur KI“). Die Einbettung von Learning Analytics in ein verantwortungsvolles Change-Management an Hochschulen zählt darüber hinaus zu den zentralen Aufgaben ebenso wie die Fragen nach ihrer Nachvollziehbarkeit (Ferguson und Clow 2017). Es bedarf weiterer Forschung zum Zusammenhang von Learning Analytics und Entwicklungen in der KI. Der NMC Horizon Report 2017 betrachtet KI als wichtigen lehr- und lerntechnologischen Bestandteil für den Hochschulbereich im Zeithorizont von vier bis fünf Jahren (Adams Becker et al. 2017). Da Lernen und Lehren vermehrt online stattfindet und Hochschulen zunehmend Datenmengen von studentischen Lernaktivitäten sammeln, können sich vielversprechende Perspektiven von Learning Analytics und KI ergeben, die es beispielsweise im Sinne des personalisierten Lernens weiter zu verfolgen, zu erforschen und zu entwickeln gilt. 


\section{Literatur}

Adams Becker, S.; Cummins, M.; Davis, A.; Freeman, A.; Hall Glesinger, C.; Anathanarayanan, V. (2017): NMC Horizon Report 2017 Higher Education Edition. Austin, Texas: The New Media Consortium. Online verfügbar unter http://cdn.nmc.org/media/2017-nmchorizon-report-he-EN.pdf, zuletzt geprüft am 05.05.2017.

Arnold, K. (2010): Signals: Applying academic analytics: EDUCAUSE Quarterly (33).

Arnold, K.; Pistilli, M. D. (Hrsg.) (2012): Course signals at Purdue: using learning analytics to increase student success. 2nd International Conference on Learning Analytics and Knowledge. Vancouver, BC, Canada, 29th-May 2nd. New York.

BMBF (2012): Perspektive MINT. Wegweiser für MINT-Förderung und Karrieren in Mathematik, Informatik, Naturwisenschaften und Technik. Online verfügbar unter https://www. bmbf.de/pub/perspektive_mint.pdf, zuletzt geprüft am 04.06.18.

Cormier, D.; Siemens, G. (2010): The open course. Through the open door: Open courses as research, learning and engagement. In: EDUCAUSE Review 45 (4), S. 30-39. Online verfügbar unter https://oerknowledgecloud.org/sites/oerknowledgecloud.org/files/ ERM1042[1].pdf, zuletzt geprüft am 04.06.18.

Elias, Tanya (2011): Learning Analytics: Definitions, Processes and Potential. Online verfügbar unter https://landing.athabascau.ca/file/download/43713, zuletzt geprüft am 05.06.2018.

Ferguson, R.; Clow, D. (Hrsg.) (2017): Where is the evidence? A call to action for learning analytics. LAK '17 Proceedings of the Seventh International Learning Analytics \& Knowledge Conference. the Seventh International Learning Analytics \& Knowledge Conference. Vancouver, BC, Canada, March 13-17: ACM.

Florida State University (2018): How do I track student activity in my course? (671). Online verfügbar unter https://support.campus.fsu.edu/kb/article/671-how-do-i-track-studentactivity-in-my-course/, zuletzt geprüft am 05.06.2018.

Greller, W.; Drachsler, H. (2012): Translating learning into numbers: A generic framework for learning analytics. In: Educational Technology \& Society 15 (3), S. 42-57. Online verfügbar unter https://pdfs.semanticscholar.org/d1bd/219962defaeb326c3b51fb4fb1086c5b7b28. pdf, zuletzt geprüft am 04.06.2018.

Heublein, U., Ebert, J., Hutzsch, C., Isleib, S., König, R., Richter, J., Woisch, A. (2017).

Zwischen Studienerwartungen und Studienwirklichkeit, Ursachen des Studienabbruchs, beruflicher Verbleib der Studienabbrecherinnen und Studienabbrecher und Entwicklung der Studienabbruchquote an deutschen Hochschulen. Hannover: DZHW.

HijonmR.; Carlos, R .: E-Learning platforms analysis and development of students tracking functionality. In: Proceedings of the 18th World Conference on Educational Multimedia, Hypermedia \& Telecomunications, S. 2823-2828.

Ifenthaler, Dirk (2015): Learning Analytics in: The SAGE encyclopedia of educational technology. 2, S. 447-451. 
Ifenthaler, Dirk; Mah, Dana-Kristin; Yau, Jane Yin-Kim (2017): Studienerfolg mittels Learning Analytics. e-teaching.org. Online verfügbar unter https://www.e-teaching.org/etresources/ pdf/erfahrungsbericht_2017_ifenthaler-et-al_studienerfolg-mittels-learning-analytics.pdf, zuletzt geprüft am 05.06.18.

Ifenthaler, Dirk; Schuhmacher, Clara (2016): Learning analytics im Hochschulkontext. In: WIST 45 (4), S. 176-181. DOI: 10.15358/0340-1650-2016-4-176.

Ifenthaler, Dirk; Widanapathirana, Chathuranga; Springer. (2014): Development and Validation of a Learning Analytics Framework. Two Case Studies Using Support Vector Machines. In: Technology, knowledge and Learning 19 (1-2), S. 221-240. DOI: 10.1007/ s10758-014-9226-4.

Ionica, Lavina (2016): Learning Analytics in der Hochschullehre. Hg. v. Hochschulforum Digitalisierung. Online verfügbar unter https://hochschulforumdigitalisierung.de/de/blog/ learning-analytics-hochschullehre, zuletzt geprüft am 30.05.2018.

Johnson, L.; Adams Becker, S.; Cummins, M.; Estrada, V.; Freeman, A.; Hall, C. (2016): NMC Horizon Report: 2016 Higher Education Edition. Hamburg: Multimedia Kontor Hamburg. Online verfügbar unter https://www.mmkh.de/fileadmin/dokumente/Publikationen/2016nmc-horizon-report-he-DE.pdf, zuletzt geprüft am 04.06.18.

Jülicher, Tim (2015): Big Data in der Bildung. Learning Analytics, Educational Data Mining und Co. In: ABIDA-Dossier.

Kellen, V.; Recktenwald, A.; Burr, S. (2013): Applying Big Data in Higher Education: A Case Study. 13. Aufl. Hg. v. Cutter Consortium (8). Online verfügbar unter https://www.cutter. com/article/applying-big-data-higher-education-case-study-400836, zuletzt geprüft am 05.06.2018.

Leitner, Philipp; Ebner, Martin; Erpenbeck, John; Sauter, Werner (2017): Learning Analytics in Hochschulen. In: Handbuch Kompetenzentwicklung im Netz. Bausteine einer neuen Lernwelt. Stuttgart: Schäffer-Poeschel Verlag, S. 371-383.

Loser, Kai-Uwe (2016): Pro und Contra: Positionen zu Learning Analytics. Hg. v. e-teaching. org. Online verfügbar unter https://www.e-teaching.org/community/meinung-old/pro_ con_learning_analytics/index_html, zuletzt aktualisiert am 17.06.2016, zuletzt geprüft am 30.05.2018.

Mah, Dana-Kristin (2016): Learning Analytics and Digital Badges. Potential Impact on Student Retention in Higher Education. In: Technology, knowledge and Learning 21 (3). New York: Springer. Online verfügbar unter http://dx.doi.org/10.1007/s10758-016-92868, zuletzt geprüft am 22.06.2018.

Merceron, Agathe; Bilkstein, Paulo; Siemens, George (2015): Learning Analytics: From Big Data to Meaningful Data. In: Journal of Learning Analytics 2 (3), S. 4-8. Online verfügbar unter https://files.eric.ed.gov/fulltext/EJ1127050.pdf, zuletzt geprüft am 05.06.18.

Nguyen, Quan; Tempelaar, Dirk; Rienties, Bart; Giesbers, Bas (2016): What learning analytics based prediction models tell us about feedback preferences of students. In: The Quarterly Review of Distance Education Band 17 (Ausgabe 3), S. 13-33. Online verfügbar unter 
https://www.researchgate.net/publication/309232936_What_learning_analytics_based_ prediction_models_tell_us_about_feedback_preferences_of_students, zuletzt geprüft am 22.6.18.

Niemann, Julia; Neu-Clausen, Maike; Hoffmann, Rüdiger (2014): Die Mentoringprogramme der Fakultät für Bildungswissenschaften. Projektbericht 2011-2014. Hg. v. Universität Duisburg-Essen, Fakultät für Bildungswissenschaften. Online verfügbar unter https:// www.uni-due.de/imperia/md/images/zfh/mentoring-tutorien/bildungswissenschaften_m\% C3\%A4rz_2015.pdf, zuletzt geprüft am 22.6.18.

Panke, Stefanie (2010): 'Web XXO' - Week Three of PLENK2010 on Emerging Technologies. In: etcjournal (a journal for educational technology \& change). Online verfügbar unter https://etcjournal.com/2010/09/29/web-xxo-\%e2\%80\%93-week-three-of-plenk2010-onemerging-technologies/, zuletzt geprüft am 22.06.2018.

Pardo, Abelardo; Siemens, George; Wiley-Blackwell (2014): Ethical and Privacy Principles for Learning Analytics. In: Br J Educ Technol 45 (3), S. 438-450. Online verfügbar unter http:// dx.doi.org/10.1111/bjet.12152, zuletzt geprüft am 22.06.2018.

Pistilli, Matthew D.; Arnold, Kimberly E. (2010): In practice. Purdue Signals: Mining real-time academic data to enhance student success. In: About Campus 15 (3), S. 22-24. DOI: 10.1002/abc.20025.

Scheffel, Maren; Drachsler, Hendrik; Specht, Marcus (Hrsg.) (2015): Developing an evaluation framework of quality indicators for learning analytics. the Fifth International Conference. Poughkeepsie, New York, 16.03.2015 - 20.03.2015. New York, New York, USA: ACM Press.

Sclater, Niall; Mullan, J. (2017): Learning analytics and student success - assessing the evidence. Hg. v. Jisc. Online verfügbar unter http://repository.jisc.ac.uk/6560/1/learninganalytics_and_student_success.pdf, zuletzt geprüft am 22.06.2018.

Sclater, Niall; Peasgood, A.; Mullan, J. (2016): Learning Analytics in Higher Education. A review of UK and international practices. Full report. Hg. v. Jisc. Jisc. Bristol. Online verfügbar unter https://www.jisc.ac.uk/sites/default/files/learning-analytics-in-he-v3.pdf, zuletzt geprüft am 22.06.2018.

Sin, Katrina; Muthu, Loganathan (2015): Application of Big Data in educational Data Mining and Learning Analytics. A literature review. In: Journal of Soft Computing: Special Issue on Soft Computing Models for Big Data 5 (4), S. 1035-1049. Online verfügbar unter http:// ictactjournals.in/paper/IJSC_V5_I4_paper6_1035_1049.pdf, zuletzt geprüft am 22.06.2018.

Slade, Sharon; Prinsloo, Paul (2013): Learning Analytics. Ethical Issues and Dilemmas. In: American Behavioral Scientist 57 (10), S. 1510-1528. DOI: 10.1177/0002764213479366.

Toetenel, Lisette; Rienties, Bart (2016): Analysing 157 learning designs using learning analytic approaches as a means to evaluate the impact of pedagogical decision-making. In: British Journal of Educational Technology Volume 47 (Issue 5), S. 981-992. Online verfügbar 
unter https://onlinelibrary.wiley.com/doi/pdf/10.1111/bjet.12423, zuletzt geprüft am 22.06.2018

Verbert, Katrien; Duval, Erik; Klerkx, Joris; Govaerts, Sten; Santos, José Luis (2013): Learning Analytics Dashboard Applications. In: American Behavioral Scientist 57 (10), S. 1500 1509. DOI: $10.1177 / 0002764213479363$.

Zhang, H.; Almeroth, K. (2010): Moodog: Tracking Student Activity in Online Course Management Systems. In: Journal of Interactive Learning Research 21 (3), S. 407-429.

\section{(c) (1)}

Dieses Kapitel wird unter der Creative Commons Namensnennung 4.0 International Lizenz http://creativecommons.org/licenses/by/4.0/deed.de) veröffentlicht, welche die Nutzung, Vervielfältigung, Bearbeitung, Verbreitung und Wiedergabe in jeglichem Medium und Format erlaubt, sofern Sie den/die ursprünglichen Autor(en) und die Quelle ordnungsgemäß nennen, einen Link zur Creative Commons Lizenz beifügen und angeben, ob Änderungen vorgenommen wurden.

Die in diesem Kapitel enthaltenen Bilder und sonstiges Drittmaterial unterliegen ebenfalls der genannten Creative Commons Lizenz, sofern sich aus der Abbildungslegende nichts anderes ergibt. Sofern das betreffende Material nicht unter der genannten Creative Commons Lizenz steht und die betreffende Handlung nicht nach gesetzlichen Vorschriften erlaubt ist, ist für die oben aufgeführten Weiterverwendungen des Materials die Einwilligung des jeweiligen Rechteinhabers einzuholen. 